\title{
Witten effect in a crystalline topological insulator
}

\author{
G. Rosenberg and M. Franz \\ Department of Physics and Astronomy, University of British Columbia, Vancouver, BC, Canada V6T $1 Z 1$
}

(Dated: October 25, 2018)

\begin{abstract}
It has been noted a long time ago that a term of the form $\theta\left(e^{2} / 2 \pi h\right) \mathbf{B} \cdot \mathbf{E}$ may be added to the standard Maxwell Lagrangian without modifying the familiar laws of electricity and magnetism. $\theta$ is known to particle physicists as the 'axion' field and whether or not it has a nonzero expectation value in vacuum remains a fundamental open question of the Standard Model. A key manifestation of the axion term is the Witten effect: a unit magnetic monopole placed inside a medium with $\theta \neq 0$ is predicted to bind a (generally fractional) electric charge $-e(\theta / 2 \pi+n)$ with $n$ integer. Here we conduct a first test of the Witten effect based on the recently established fact that the axion term with $\theta=\pi$ emerges naturally in the description of the electromagnetic response of a new class of crystalline solids called topological insulators - materials distinguished by strong spinorbit coupling and non-trivial band structures. Using a simple physical model for a topological insulator we demonstrate the existence of a fractional charge bound to a monopole by an explicit numerical calculation. We also propose a scheme for generating an 'artificial' magnetic monopole in a topological insulator film, that may be used to facilitate the first experimental test of Witten's prediction.
\end{abstract}

\section{AXIONS}

The idea of the axion was introduced in 1977 by Peccei and Quinn ${ }^{1}$ as a means to solve what is known as the 'strong CP problem' in the physics of strong interactions. The strong $\mathrm{CP}$ problem, the details of which are quite subtle, has to do with the vacuum structure of Quantum Chromodynamics. In simple physical terms it can be stated as a question: Why is the electric dipole moment of the neutron (currently unobserved) so small? The Standard Model predicts a value for the neutron dipole moment $\left|\mathbf{d}_{n}\right| \sim 10^{-16} \theta$ e cm, with $\theta$ of order unity, that should be readily measurable. Peccei-Quinn's solution promotes $\theta$ to a dynamical field describing a new elementary particle, the axion, whose vacuum expectation value has relaxed to a very small value, explaining the smallness of $\left|\mathbf{d}_{n}\right|$. The actual value of $\theta$, and the validity of the Peccei-Quinn solution and its variants $\$ 213$ remain open questions of considerable importance to fundamental physics. The axion is also believed to be a viable candidate for the elusive dark matter that comprises the majority of matter in our universe ${ }^{4}$ and is subject to active experimental searches $\left[\frac{5] 6}{6}\right.$

In a remarkable development axion electrodynamics has recently emerged as a key tool in the description of crystalline solids called strong topological insulators (STIs). These three-dimensional time-reversal invariant (TRI) materials possess anomalous band structures characterized by a $\mathrm{Z}_{2}$-valued topological invariant ${ }^{788}$ This invariant, called $\nu_{0}$, counts the number of topologically protected gapless surface states (modulo 2). A non-zero invariant means that the surface of such an insulator will be metallic. This behavior has been predicted to occur $9 / 10$ and subsequently experimentally discovered 1113 in several 3-dimensional solids such as $\mathrm{Bi}_{1-x} \mathrm{Sb}_{x}$ alloys and $\mathrm{Bi}_{2} \mathrm{Se}_{3}, \mathrm{Bi}_{2} \mathrm{Te}_{3}$ crystals. More recently it has been realized, $\frac{14 \mid 15}{15}$ remarkably, that the electromagnetic re- sponse of a STI is characterized by the axion term

$$
\Delta \mathcal{L}_{\text {axion }}=\theta\left(\frac{e^{2}}{2 \pi h}\right) \mathbf{B} \cdot \mathbf{E}
$$

with $\theta=\pi$, the only non-zero value permitted by the time-reversal symmetry. When the time-reversal symmetry is broken, e.g. in a crystal showing weak magnetism, $\theta$ can acquire an arbitrary value. Fluctuations in the magnetic order parameter then act as a dynamical axion field and can be thought of as emergent axion particles. 17 Thus, aside from possible practical applications, crystalline solids with topologically non-trivial band structures have the potential to provide tabletop laboratories for the testing and exploration of fundamental physical paradigms.

A fundamental property of the axion medium is the Witten effect: $\frac{18}{10}$ the quantum theory, a magnetic monopole of unit strength (i.e. projecting magnetic flux $\Phi_{0}=h c / e$ ) immersed in an axion medium must carry electric charge $-e(\theta / 2 \pi+n)$ with $n$ integer. This effect, although theoretically well established, has never been experimentally tested because until now both a suitable axion medium and the means to produce a magnetic monopole have been lacking. In this study we demonstrate how the connection between the axion

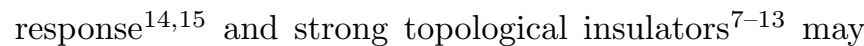
serve to overcome both obstacles. We remark that a 1D realization of the Witten effect in antiferromagnetic spin chains was proposed a long time ago $\frac{19}{19}$ Here we furnish the first concrete physical example of the Witten effect in $3 \mathrm{D}$ by modeling a STI with a magnetic monopole inserted in its bulk. We show that the monopole binds a fractional charge $\pm e / 2$ consistent with Witten's prediction! 18 We then discuss possible ways to overcome the second obstacle by creating an emergent magnetic monopole in a topological insulator. This can be achieved by exploiting the degrees of freedom associated with a vortex in the exciton condensate that may emerge in a thin film topo- 
logical insulator under external bias. ${ }^{20}$ We conclude that the prospects for experimental verification of the Witten effect in a tabletop experiment using a STI appear promising.

\section{MONOPOLE AND THE WITTEN EFFECT}

We start with a brief overview of the Witten effect. Although the effect is quantum-mechanical in nature its essence can be understood by studying the classical Maxwell's equations modified in the presence of $\Delta \mathcal{L}_{\text {axion }}$. The axion term revises both Gauss' law and Ampère's law by adding extra source terms, 21

$$
\begin{aligned}
\nabla \cdot \mathbf{E} & =\rho-\frac{\alpha}{4 \pi^{2}} \nabla \theta \cdot \mathbf{B}, \\
\nabla \times \mathbf{B} & =\frac{\partial \mathbf{E}}{\partial t}+\mathbf{j}+\frac{\alpha}{4 \pi^{2}}\left(\nabla \theta \times \mathbf{E}+\frac{\partial \theta}{\partial t} \mathbf{B}\right),
\end{aligned}
$$

where $\alpha=e^{2} / \hbar c$ is the fine structure constant and $\rho, \mathbf{j}$ are the electric charge density and current, respectively. We observe that for uniform, constant $\theta$, Eqs. (2) and (3) revert to the familiar Maxwell's equations, consistent with the notion that $\Delta \mathcal{L}_{\text {axion }}$ can be written as a total derivative in this case. An important related property 21 is the periodicity under $\theta \rightarrow \theta+2 \pi n$ of the axion action $\mathcal{S}_{\text {axion, }}$ implying that $\theta$ can be chosen from the interval $[0,2 \pi)$.

Now consider a unit monopole, $\nabla \cdot \mathbf{B}=\Phi_{0} \delta(\mathbf{r})$, placed at the origin, in a medium initially characterized by $\theta=0$. We wish to understand what happens when we turn on $\theta$ as a function of time (but keep it uniform in space). To this end we set $\nabla \theta=0$ and $\mathbf{j}=0$ (no currents in vacuum) and take the divergence of Eq. (3) to obtain

$$
\nabla \cdot \frac{\partial \mathbf{E}}{\partial t}+\frac{\alpha}{4 \pi^{2}} \frac{\partial \theta}{\partial t} \nabla \cdot \mathbf{B}=0 .
$$

We see that an electric field is generated in this process. Integrating Eq. (4) over space and time, we find that this field can be thought of as originating from a point electric charge $Q$ located at the origin with magnitude

$$
Q=-\frac{\alpha}{4 \pi^{2}} \Phi_{0} \Delta \theta=-\frac{\Delta \theta}{2 \pi} e,
$$

where $\Delta \theta$ is the net change in $\theta$ and we assumed that there was no initial electrical charge bound to the monopole, as should be the case for a charge-conjugation and parity $(\mathrm{CP})$ invariant theory $\frac{18}{18}$ with $\theta=0$. In a topological insulator $\Delta \theta=\pi$, thus one expects a magnetic monopole to bind fractional charge

$$
Q=-e\left(\frac{1}{2}+n\right)
$$

The integer $n$ accounts for the possibility of binding extra electrons, which can always occur - only the fractional part of $Q$ is non-trivial.

\section{TOPOLOGICAL INSULATOR AS AN AXION MEDIUM}

We now specify our model for a topological insulator and show that it indeed possesses the axion term. In order to minimize computational difficulties we consider a very simple model, inspired by Ref. 22 , with electrons hopping on the cubic lattice with two orbitals per site, denoted as $c$ and $d$. The Hamiltonian $H=H_{\mathrm{SO}}+H_{c d}$, consists of a spin dependent part, with hopping between neighboring sites of the lattice,

$$
H_{\mathrm{SO}}=i \lambda \sum_{j, \mu} \Psi_{j}^{\dagger} \tau_{z} \sigma_{\mu} \Psi_{j+\mu}+\text { h.c. },
$$

where $\Psi_{j}=\left(c_{j \uparrow}, c_{j \downarrow}, d_{j \uparrow}, d_{j \downarrow}\right)^{T}, j$ labels sites of the cubic lattice, $\tau_{\mu}$ and $\sigma_{\mu}$ are Pauli matrices in orbital and spin space, respectively, $\mu=x, y, z$, and the spin-independent terms that connect the two orbitals,

$$
H_{c d}=\epsilon \sum_{j} \Psi_{j}^{\dagger} \tau_{x} \Psi_{j}-t \sum_{\langle i j\rangle} \Psi_{i}^{\dagger} \tau_{x} \Psi_{j}+\text { h.c. }
$$

Although the model specified by Eqs. (7) and (8) probably does not describe any real solid, it is physical in that it is local in space and preserves time reversal and inversion symmetries. We show below that for a range of parameters $(\lambda, \epsilon, t)$ it represents a topological insulator, and therefore can be adiabatically deformed into any

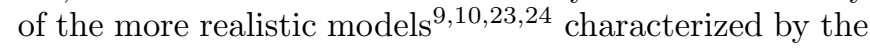
same topological invariants. Any physical property that depends only on the topological invariants, such as the electrical charge bound to a monopole, can thus be calculated in the present model and the result will remain applicable to any topological insulator in the same topological class. We note that a similar model was used in Refs. 10[17/25.

Our Hamiltonian has a simple representation in momentum space, $H=\sum_{\mathbf{k}} \Psi_{\mathbf{k}}^{\dagger} \mathcal{H}_{\mathbf{k}} \Psi_{\mathbf{k}}$ with

$$
\mathcal{H}_{\mathbf{k}}=-2 \lambda \sum_{\mu} \tau_{z} \sigma_{\mu} \sin k_{\mu}+\tau_{x} m_{\mathbf{k}},
$$

and $m_{\mathbf{k}}=\epsilon-2 t \sum_{\mu} \cos k_{\mu}$. The spectrum of excitations has two doubly degenerate bands,

$$
E_{\mathbf{k}}= \pm \sqrt{4 \lambda^{2}\left(\sin ^{2} k_{x}+\sin ^{2} k_{y}+\sin ^{2} k_{z}\right)+m_{\mathbf{k}}^{2}}
$$

In the limit $\epsilon, t \rightarrow 0$ the bands touch at 8 non-equivalent Dirac points located at $\boldsymbol{\Gamma}_{\ell=\left(n_{x} n_{y} n_{z}\right)}=\pi\left(n_{x}, n_{y}, n_{z}\right)$ with $n_{\mu}=0,1$. These $\boldsymbol{\Gamma}_{\ell}$ also coincide with the 8 time-reversal invariant momenta ${ }^{9}$ (TRIM). When $\epsilon, t$ are small but non-zero, the low-energy excitations of the system can be described in terms of 8 massive Dirac Hamiltonians

$$
\mathcal{H}_{\mathbf{k}}^{\ell}=\sum_{\mu} \tau_{z} \sigma_{\mu} v_{\mu}^{\ell} k_{\mu}+\tau_{x} m_{\ell}
$$

obtained by a straightforward expansion of $\mathcal{H}_{\mathbf{k}}$ to linear order in momentum in the vicinity of $\boldsymbol{\Gamma}_{\ell}$. Here 
TABLE I: $Z_{2}$ indices $\left(\nu_{0} ; \nu_{1} \nu_{2} \nu_{3}\right)$ calculated according to Ref. 9 for our model insulator at half filling and the corresponding values of the axion parameter $\theta . \nu_{0}$ is the important 'strong' invariant while $\nu_{i=1,2,3}$ are the so called 'weak' invariants $\frac{788}{7}$ which do not play a role in the present study but we list them here for completeness. It is assumed that $\lambda, t>0$. WTI denotes a 'weak' topological insulator.

\begin{tabular}{clcc}
\hline Parameters & $Z_{2}$ class & Insulator type & Axion $\theta$ \\
\hline$|\epsilon|>6 t$ & $(0 ; 000)$ & trivial & 0 \\
$-6 t<\epsilon<-2 t$ & $(1 ; 111)$ & STI & $\pi$ \\
$-2 t<\epsilon<2 t$ & $(0 ; 111)$ & WTI & 0 \\
$2 t<\epsilon<6 t$ & $(1 ; 000)$ & STI & $\pi$ \\
\hline
\end{tabular}

$v_{\mu}^{\ell} \equiv-2 \lambda(-1)^{n_{\mu}}$ are the Cartesian components of the Dirac velocities at $\boldsymbol{\Gamma}_{\ell}$ and $m_{\ell} \equiv m_{\boldsymbol{\Gamma}_{\ell}}$ are the corresponding Dirac masses. The Hamiltonians $\mathcal{H}_{\mathbf{k}}^{\ell}$ show spectra

$$
E_{\mathbf{k}}^{\ell}= \pm \sqrt{4 \lambda^{2} k^{2}+m_{\ell}^{2}} .
$$

The system described by Hamiltonian (9) is inversion symmetric and we can thus employ the method devised in Ref. 9 to determine the topological class of its insulating phases when the negative-energy bands are filled with electrons. This straightforward method requires computing the eigenvalues of the parity operator at the 8 TRIM for the occupied bands. We find four distinct phases, depending on parameters $\epsilon$ and $t$, two of which are STI. The complete results are listed in Table I.

According to general considerations ${ }^{14115}$ the STI phases should exhibit the axion term with $\theta=\pi$. We evaluate $\theta$ for our model using the non-abelian Berry connection $\mathcal{A}_{i}^{\alpha \beta}=-i\left\langle\alpha \mathbf{k}\left|\partial_{i}\right| \beta \mathbf{k}\right\rangle$ and the formula ${ }^{14 \mid 15}$

$$
\theta=\frac{1}{4 \pi} \int_{\mathrm{BZ}} d^{3} k \epsilon^{i j k} \operatorname{Tr}\left[\mathcal{A}_{i} \partial_{j} \mathcal{A}_{k}+\frac{2 i}{3} \mathcal{A}_{i} \mathcal{A}_{j} \mathcal{A}_{k}\right],
$$

where $|\beta \mathbf{k}\rangle$ is an eigenstate of $\mathcal{H}_{\mathbf{k}}$, the trace extends over occupied states and $\partial_{i} \equiv \partial / \partial k_{i}$. The integral indicated in Eq. (13) is generally difficult to evaluate and numerical methods must be used to obtain $\theta$ for an arbitrary band structure. For a model in which the band touching is described by Dirac Hamiltonians, however, a simple analytical evaluation of Eq. (13) is possible by noticing that in the limit of a small Dirac mass the entire contribution to the integral comes from the Dirac points. We show in the Appendix that each Dirac point contributes

$$
\theta^{\ell}=-\frac{\pi}{2} \operatorname{sgn}\left(v_{x}^{\ell} v_{y}^{\ell} v_{z}^{\ell} m_{\ell}\right)
$$

to the total $\theta=\sum_{\ell} \theta^{\ell} \bmod 2 \pi$. Although Eq. 14 has been derived for the case $\left|m_{\ell}\right| \ll|\lambda|$ we expect it to be more generally valid for all TRI Hamiltonians that can be deformed into the form of Eq. (9). This is because the value of $\theta$ in a TRI insulator is quantized and can only change when a band crossing closes the gap. As long as band crossings occur only at $\boldsymbol{\Gamma}_{\ell}$ points and are described by Dirac Hamiltonians, $\theta$ will be determined by Eq. 14, even when $\left|m_{\ell}\right|$ is not small.
It is easy to see that Eq. 14 gives the anticipated result for our model. Consider first the situation when $\epsilon>6 t>0$ which according to Table I is a trivial insulator. In this case all 8 masses $m_{\ell}$ are positive. Since four of the products $v_{x}^{\ell} v_{y}^{\ell} v_{z}^{\ell}$ are positive and four are negative Eq. (14) gives $\theta=0$, as expected. Now suppose we tune $\epsilon$ so that its value drops below $6 t$. This reverses the sign of a single Dirac mass at $\ell=(0,0,0)$. The corresponding $\theta^{\ell}$ also reverses sign and we obtain $\theta=\pi$, as expected for a STI. For all other cases the values of $\theta$ are listed in Table I. These results confirm the one-toone correspondence between the $Z_{2}$ invariant $\nu_{0}$ and the axion parameter $\theta$ expressed by $\theta=\pi \nu_{0}$, as expected on very general grounds. 16

\section{CHARGE BOUND TO A MONOPOLE}

We now consider a magnetic monopole in the interior of a STI. We model this situation by the Hamiltonian $H$ defined by Eqs. (7) and (8) with a monopole positioned at the center of a cubic unit cell. The magnetic field of the monopole couples to both the electron charge and the electron spin through the orbital and Zeeman couplings, respectively. The form of the orbital coupling is dictated by gauge invariance and is thus universal; in our lattice model it is implemented by the Peierls substitution, which attaches factors $e^{i \vartheta_{i j}}$ to all hopping terms connecting sites $i$ and $j$. Here $\vartheta_{i j}=\left(2 \pi / \Phi_{0}\right) \int_{i}^{j} \mathbf{A} \cdot d \mathbf{l}$ and $\mathbf{A}$ is the magnetic vector potential. The Zeeman coupling is of the form $-g \mu_{B} \mathbf{B} \cdot \mathbf{S} / \hbar$ where $\mu_{B}=e \hbar / 2 m_{e} c$ is the Bohr magneton and $\mathbf{S}$ denotes the electron spin. For free electrons $g$ is close to 2 but in solids the effective $g$ can be substantially larger. The Zeeman coupling thus leads to an additional term in the Hamiltonian,

$$
H_{Z}=-g \mu_{B} \frac{1}{2} \sum_{j} \mathbf{B}_{j} \cdot\left(\Psi_{j}^{\dagger} \sigma \Psi_{j}\right),
$$

where $\mathbf{B}_{j}$ is the magnetic field at site $j$ of the lattice. This term is non-universal and its importance will depend on the ratio of $g \mu_{B}|\mathbf{B}|$ to the other relevant energy scales in the Hamiltonian set by $\lambda, \epsilon$ and $t$.

We solve the Hamiltonian $H=H_{\mathrm{SO}}+H_{c d}+H_{Z}$ in a cube containing $L^{3}$ sites by exact numerical diagonalization. The monopole is placed inside the central unit cell (at the origin), so that the magnetic field of the monopole is $\mathbf{B}=\left(\Phi_{0} / 4 \pi r^{2}\right) \hat{r}$. We choose a gauge in which the system retains the four-fold rotational symmetry around the $z$ axis,${ }^{26} \mathbf{A}=-\Phi_{0}(1+\cos \theta) \nabla \varphi$, with $(\theta, \varphi)$ the spherical angles. Exploiting this symmetry we are able to simulate system sizes up to $L=20$, which requires diagonalizing a complex valued Hermitian matrix of size $\frac{1}{4}\left(4 \times 20^{3}\right)=8,000$. In order to calculate the charge density at half filling we require knowledge of all the occupied eigenstates.

We diagonalize the Hamiltonian, once with the magnetic monopole and once without, obtaining charge densities $\rho_{1}$ and $\rho_{0}$, respectively. The monopole-induced 

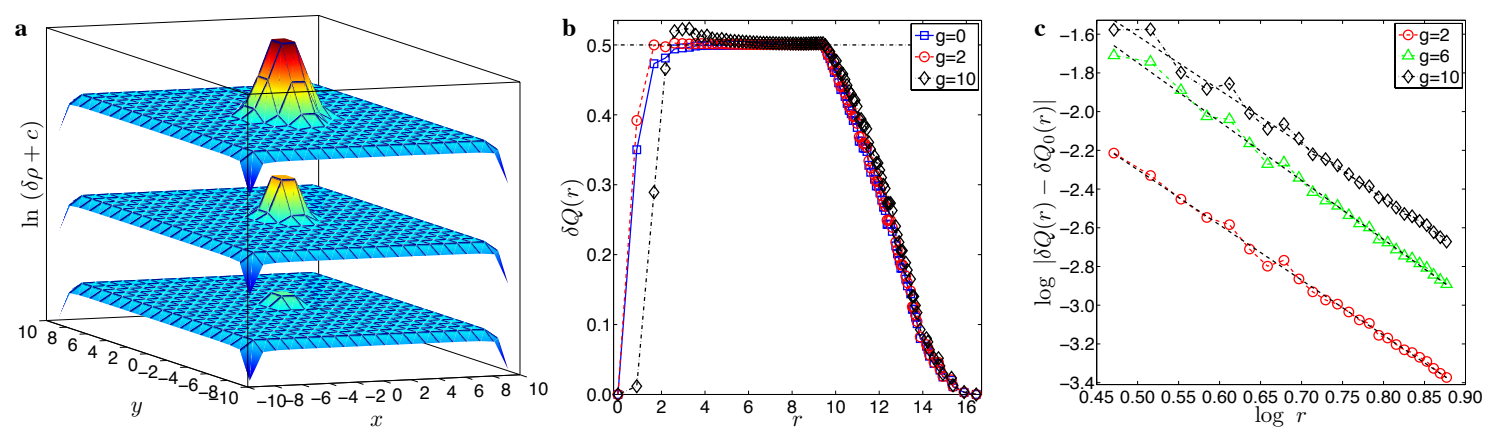

FIG. 1: (Color online) Charge density in our model TI on the cube-shaped lattice with $20^{3}$ sites with a unit monopole at its center, with parameters $t=\lambda, \epsilon=4 t$, leading to a bulk gap $\Delta=4 t$. (a) Charge density $\delta \rho$ of the three closest layers below the monopole, for $g=0$. (b) The excess charge $\delta Q(r)$ (in units of $e$ ) for different Zeeman coupling $g$. The knee feature seen at $r=10$ corresponds to the radius at which the sphere used to calculate $\delta Q(r)$ first touches the system boundary. (c) Log-log plot of $\delta Q_{g}-\delta Q_{0}$ showing the power-law approach $\sim r^{-\alpha}$ of the accumulated charge to its assymptotic value of $1 / 2$. The least-square fit yields exponents $\alpha=2.85,3.04,2.79$ for $g=2,6,10$, respectively. We attribute the deviations of the numerically determined exponent $\alpha$ from the expected value of 3 to the finite size effect.

charge density $\delta \rho=\rho_{0}-\rho_{1}$ is plotted in Fig. 17. To determine the total charge bound to the monopole we calculate the excess accumulated charge in a sphere of radius $r$ centered on the monopole, $\delta Q(r)=\sum_{\left|\mathbf{r}_{i}\right|<r} \delta \rho\left(\mathbf{r}_{i}\right)$. We find (Fig. 1 1 b) that it saturates at $-e / 2$ to within 4 significant digits, comparable to the accuracy of our numerics. For $g=0$ we find two localized zero modes, one at the monopole and one on the surface. Fractional charge bound to the monopole can be understood in this case by appealing to the standard arguments28|29] developed originally to describe charge fractionalization in polyacetylene $\stackrel{30}{30}$ Briefly, when a topological defect (such as a domain wall in polyacetylene) produces a localized zero mode inside the gap in a particle-hole symmetric system, one can show that the spectral weight of the state contains equal contributions from the valence and the conduction bands. Thus, the valence band shows a net deficit of half a state in the vicinity of the defect. This translates into the defect carrying fractional charge $\pm e / 2$, the sign depending on whether the zero mode is empty or occupied.

Like in polyacetylene we find the saturation of charge to be exponential $\sim \exp (-r / \xi)$, where $\xi \sim 1 / \Delta$ and $\Delta$ is the bulk gap.

When $g>0$, the Zeeman coupling causes changes in the charge distribution near the monopole but the total accumulated charge remains quantized at $-e / 2$. In this case there are no exact zero modes in the spectrum and $\delta Q(r)$ approaches $e / 2$ as a power law with the exponent close to -3 (Fig. 11.).

The power-law dependence can be understood as follows. The Zeeman term acts as an additional timereversal breaking field which modifies the value of axion $\theta$ away from $\pi$ close to the monopole. This causes non-vanishing $\nabla \theta$ and thus, according to Eq. 22), additional contribution to the effective charge density. The simplest assumption, $\delta \theta \sim \mathbf{B}^{2}$, gives $\delta \rho \sim \nabla \theta \cdot \mathbf{B} \sim r^{-7}$ and $\delta Q \sim r^{-4}$, a power law but with the exponent not quite in agreement with our numerical simulation. On further reflection one realizes that in our model $\delta \theta$ cannot be proportional to $\mathbf{B}^{2}$ but rather must be proportional to its gradients. This is because in the presence of a uniform Zeeman term the system remains inversionsymmetric. Inversion symmetry dictates quantized value of $\theta=0, \pi$ even when $\mathcal{T}$ is explicitly broken! ${ }^{14115}$ Thus, non-vanishing $\delta \theta$ requires spatially varying Zeeman field. The simplest assumption satisfying these requirements is $\nabla \theta \sim \nabla^{2} \mathbf{B}$. In the vicinity of the monopole one finds $\delta \rho \sim \nabla \theta \cdot \mathbf{B} \sim r^{-6}$ and $\delta Q \sim r^{-3}$ in agreement with our numerical results.

We note that the above considerations are based on the effective axion action (1) and apply on lengthscales large compared to $\xi$. The power law tail in the fractional charge distribution for $g>0$ appears on top of a shortlengthscale structure with a roughly exponential profile that is controlled by the properties of the microscopic Hamiltonian and is thus non-universal. At the intermediate lengthscales the interplay of the two contributions can give rise to interesting structures such as the peak in $\delta Q(r)$ at $r \simeq 2.5$ seen in Fig. $1 \mathrm{~b}$ for $g=10$.

By the same method described above we have investigated spin density induced by the monopole. We find that there is no net spin $\langle\mathbf{S}\rangle$ attached to the monopole. Thus, in addition to charge fractionalization, a magnetic monopole inserted in a STI constitutes an example of spin-charge separation in three spatial dimensions. This is perhaps not surprising in view of the fact that spinorbit coupling present in the Hamiltonian (7) breaks the $\mathrm{SU}(2)$ spin symmetry and, as a result, electron spin is not a good quantum number in the model describing our system. 


\section{PROPOSAL FOR EXPERIMENTAL REALIZATION}

Although there is no known theoretical principle that prohibits the existence of fundamental magnetic monopoles in nature ${ }^{26}$ none have been observed to date despite extensive searches. ${ }^{27}$ This null observation has led to a consensus that fundamental monopoles either do not exist for some heretofore unknown reason or they are very rare in our part of the universe. In either case the observed absence of fundamental monopoles poses a challenge to the idea of experimental verification of the Witten effect using a STI. At best, one could conceive of a new scheme for possible detection of magnetic monopoles using a STI in the role of a sensor if a convenient way to detect the fractional charge could be found.

A much more promising avenue for the verification of the Witten effect is suggested by exploiting emergent instead of fundamental monopoles. A classic example of such an emergent behavior in a crystalline solid is the 2007 theoretical prediction 31 and the subsequent experimental observation $32 \sqrt{34}$ of monopoles in frustrated magnetic systems called 'spin ice', realized in certain magnetic pyrochlore compounds such as $\mathrm{Dy}_{2} \mathrm{Ti}_{2} \mathrm{O}_{7}$ or $\mathrm{Ho}_{2} \mathrm{Ti}_{2} \mathrm{O}_{7}$. Magnetic monopoles in these systems arise as elementary excitations above the collective ground state of spins and the monopole-like magnetic field configuration originates from the magnetic moments of the constituent spins. In principle, the emergent monopoles in the spin ice could be used to test the Witten effect if a compound that is simultaneously a STI and a spin ice could be identified. Unfortunately no such material is known at present although we note that STI behavior has been theoretically predicted to occur in crystals with the same pyrochlore structure ${ }^{2324}$ that underlies the spin ice behavior. It is thus possible that a suitable material will be discovered in the future.

Here we focus on a different type of emergent magnetic monopole that can arise in a thin film STI placed in a uniform external electric field. The basic idea and the feasibility of its experimental realization have been discussed in Ref. 20. Following that work we envision the simplest STI with just one gapless Dirac state per surface and the chemical potential initially tuned to the neutral point. When a strong enough electric field is applied perpendicular to the plane of the film the chemical potential undergoes a shift that is opposite in the two surfaces. This creates a small electron Fermi surface in one surface and a small hole Fermi surface in the other. The essence of the proposa ${ }^{201}$ lies in the observation that an arbitrarily weak Coulomb interaction between the surface states produces an exciton condensate, which may be viewed as a coherent fluid of electron-hole pairs drawn from the opposite surfaces. Such an exciton condensate is characterized by a complex scalar order parameter $\Phi$, which can fluctuate in space and time. In particular $\Phi=\Phi_{0} e^{i \chi}$ can contain vortices - point-like topological defects with the phase $\chi$ winding by $\pm 2 \pi$ around a vortex. It has been pointed out in Ref. 20 that to electrons in a STI such a vortex is indistinguishable from a 'planar monopole', i.e. a monopole with magnetic field radiating in the plane of the surface.

A planar monopole can be viewed as an adiabatic deformation of an ordinary monopole achieved by flattening the field lines in a cylindrically symmetric fashion. One expects that the total charge bound to the monopole via the Witten effect should be insensitive to such an adiabatic deformation and therefore a vortex in the exciton condensate should bind fractional charge $\pm e / 2$. This indeed has been argued to happen in Ref. 20 based on the Dirac equation describing the low-energy physics of the surface states in the presence of the exciton condensate. Here, taking a more general point of view, we establish the existence of the fractional charge in such a condensate by studying a planar monopole embedded inside a STI. Our calculation below does not rely on the low-energy approximation for the surface states and is insensitive to the detailed microscopic structure of the condensate. Rather, it exploits only the most fundamental property of the STI given by its nontrivial axion response.

In general, the fractional charge is expected to be robust against weak disorder that does not break TRI. Such a disorder will be present in a real sample and we model it here by adding a term

$$
H_{D}=\sum_{j} \mu_{j}^{+} \Psi_{j}^{\dagger} \Psi_{j}+\sum_{j} \mu_{j}^{-} \Psi_{j}^{\dagger} \tau_{z} \Psi_{j},
$$

to the Hamiltonian. The first term represents a paritypreserving on-site disorder (independent of the orbital), while the second term is a parity breaking disorder.

As before we solve the Hamiltonian $H=H_{\mathrm{SO}}+H_{c d}+H_{D}$, in a cube containing $L^{3}$ sites, by exact numerical diagonalization. The planar monopole projects an effective magnetic field $\mathbf{B}_{\text {eff }}=\left(\Phi_{0} / 2 \pi r\right) \delta(z) \hat{r}$ (in cylindrical coordinates) and the vector potential can be chosen as $\mathbf{A}=\left(\Phi_{0} / 2 \pi\right) \varphi \delta(z) \hat{z}$. The effective field does not couple to electron spin,20 so there is no Zeeman term in this case. The disorder coefficients $\mu_{j}^{ \pm}$ are chosen from a Gaussian distribution with standard deviation $\mu$. Note that the disorder breaks the four-fold rotational symmetry of the system, so we cannot exploit this symmetry in this case to efficiently diagonalize the Hamiltonian. Consequently we are limited to system sizes up to $L=14$. For weak disorder $\mu \ll \Delta$ the charge bound to the planar monopole remains $-e / 2$ (see Fig. 2), and for strong disorder $\mu \gg \Delta$ the charge bound is zero. Remarkably, even for fairly significant disorder (such that it generates charge density fluctuations comparable to the charge density induced by the monopole) the difference in charge density $\delta \rho$ shown in Fig. 2f is only weakly affected.

In the framework of the current proposal the key ingredient required to produce a monopole-like configuration is the exciton condensate. As explained in Ref. 20 it is difficult to reliably estimate the critical temperature $T_{E C}$ for the formation of the exciton condensate, but un- 

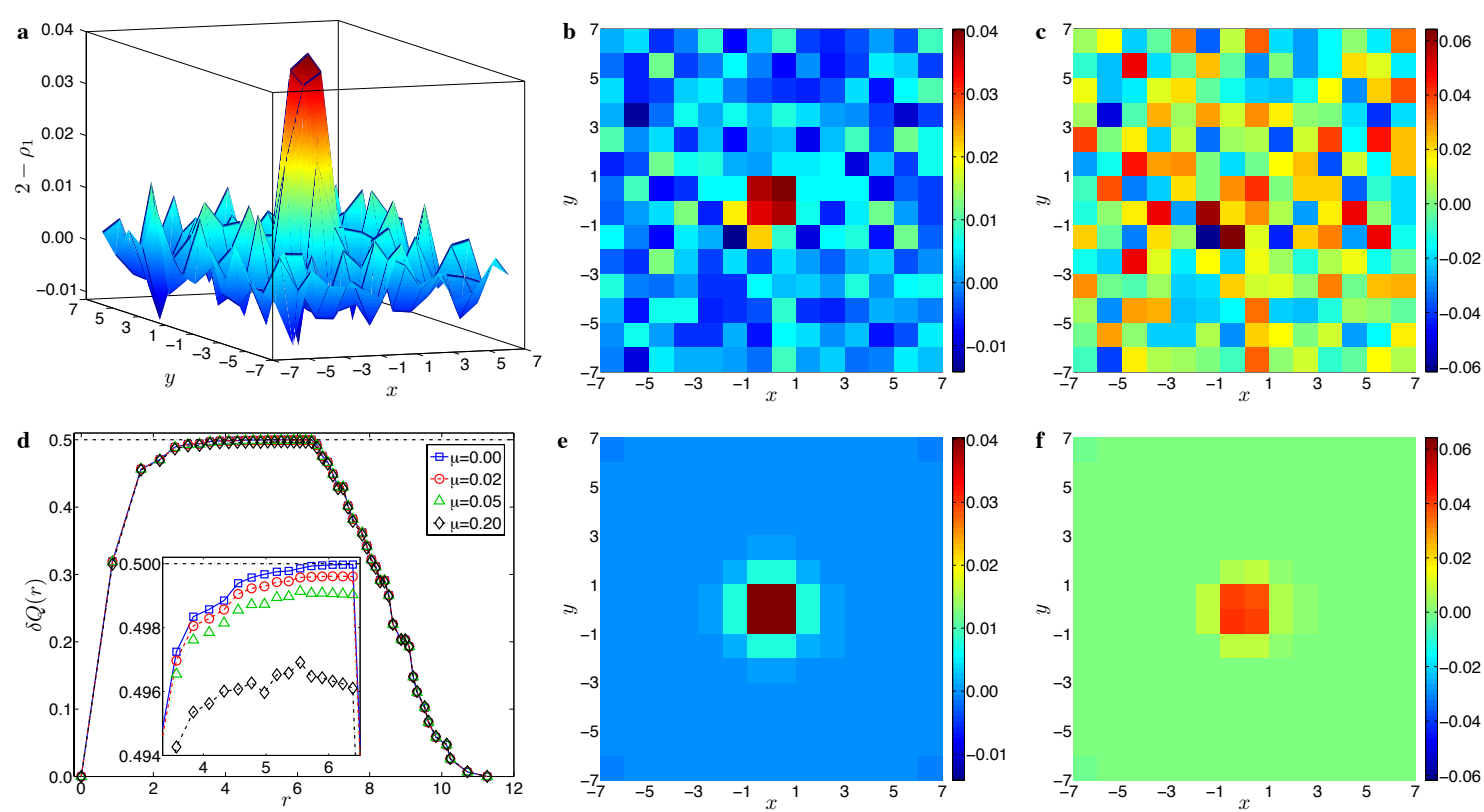

FIG. 2: (Color online) A cubic sample of a TI including disorder with a planar unit monopole at its center, size $L=14$ and parameters as in Fig. 1. In all cases shown we use the same disorder realization but vary its overall strength parametrized by $\mu$. Panels (a) and (b) show charge density $2-\rho_{1}$ for the layer just below the planar monopole for weak disorder $\mu=0.05 \Delta$, and (c) larger disorder $\mu=0.20 \Delta$. Panels (e) and (f) show the difference in charge density $\delta \rho=\rho_{0}-\rho_{1}$ for $\mu / \Delta=0.05,0.20$, respectively, for the same layer. (d) The excess charge $\delta Q(r)$ (in units of $e$ ) for different values of disorder strength $\mu$. The inset shows a close up of the saturation. At this scale a small deviation from the expected asymptotic value $1 / 2$ that increases with the disorder strength becomes visible. We attribute this deviation to the finite-size effect in our numerical calculation. This identification is supported by the fact that the deviations grow more pronounced for smaller system sizes and close to the surface. Also, it is consistent with the notion that the bound charge is localized on the length scale $\xi \sim 1 / \Delta$ which increases as the disorder reduces the spectral gap.

der optimal conditions it should be higher than it is in bilayer graphene, where the occurrence of this effect is hotly debated. Once the exciton condensate is formed, vortices can be nucleated by applying an in-plane magnetic field. Since the exciton condensate is itself insulating, the main conduction channel in this situation will be through vortices, each carrying $-e / 2$ charge. Fractional charge of the carriers then can be detected using established techniques. ${ }^{35136}$

\section{OUTLOOK AND OPEN QUESTIONS}

Predicted more than 30 years ago in the context of high-energy physics, but never before observed in a real or numerical experiment, the Witten effect is realized in a strong topological insulator. A unit magnetic monopole inserted in a model STI binds electric charge $-e / 2$ in accordance with the prediction $\frac{18}{18}$ and furnishes a rare example of charge fractionalization and spin-charge separation in 3 spatial dimensions. In the special case when the underlying system possesses particle-hole symmetry and when the Zeeman term (15) can be neglected, the appearance of fractional charge follows from the same 'zeromode' arguments that underlie charge fractionalization in a one-dimensional system of fermions coupled to a scalar field with a soliton profile $e^{28 / 29}$ as realized in dimerized polyacetylene $\stackrel{30}{30}$ In the more general case when the Zeeman term or weak disorder are present, there exist no exact zero modes in the spectrum of electrons yet the fractional charge remains precisely quantized. This reflects the more subtle topological order that underlies the axion response of a STI, which is robust against any weak perturbation that respects time reversal symmetry! $78 / 14 \mid 15$

An interesting open question is the fate of the Witten effect in the presence of magnetic disorder. Experimentally, this can be implemented by adding a small concentration of magnetic ions (such as $\mathrm{Fe}$ or $\mathrm{Mn}$ ) into a topological insulator. Although at the microscopic level $\theta$ is no longer quantized in the presence of $\mathcal{T}$-breaking perturbations, we expect its effective value, relevant to the physics at long lengthscales, to remain pinned at $\pi$ as long as the magnetic moments stay disordered. This is because the net magnetic moment in a macroscopic region containing many impurities will effectively vanish. Qualitatively, this suggests that the Witten effect may survive inclusion of a moderate concentration of magnetic dopants with randomly oriented moments. At low temperatures moments may order ferromagnetically ${ }^{[37} \mathrm{In}$ this case both $\mathcal{T}$ and the inversion symmetry are broken (the latter due to the random position of magnetic dopants) and the effective $\theta$ can acquire an arbitrary 
value. In this situation we expect a monopole to still bind fractional charge according to Eq. (6) but we leave a detailed study of this case to future investigation.

Can the Witten effect be observed experimentally in the near future? We believe that the answer is affirmative. One essential ingredient, the axion medium, is now widely available in any of the recently discovered STIs! 11-13 If an emergent monopole can be realized, exploiting the proposed exciton condensate, 20 the spin icetype physics, $31+34$ or by some other means, then the experimental challenge is reduced to designing a suitable method for the detection of fractional charge bound to the monopole. The fractional charge of elementary excitations in fractional quantum Hall fluids has been previously detected ${ }^{35 \mid 36}$ and it should be possible to adapt these methods to topological insulators. In this way, studies of crystalline quantum matter with non-trivial topological properties could help settle one of the enduring challenges of fundamental physics and provide new insights into the behavior of electrons placed in unusual situations.

\section{Acknowledgment}

The authors are indebted to I. Affleck, A.M. Essin, I. Garate, H.-M. Guo, J.E. Moore, C. Weeks, X.-L. Qi, and S.-C. Zhang for helpful discussions and correspondence. This work was supported by NSERC and CIfAR and was performed, in part, at the Aspen Center for Physics.

\section{Appendix A: Evaluation of $\theta$}

The feature that makes our model Hamiltonian (9) easy to analyze, is its matrix structure in the combined orbital/spin space, which consists of four anticommuting $4 \times 4$ matrices. Such matrices are by convention denoted $\gamma_{\mu}, \mu=0,1,2,3$ and form a representation of the Clifford algebra defined by the anticommutation relation $\left\{\gamma_{\mu}, \gamma_{\nu}\right\}=2 \delta_{\mu \nu}$. In terms of these matrices Eq. (9) can be written as

$$
\mathcal{H}_{\mathbf{k}}=\sum_{\mu} \gamma_{\mu} d_{\mu}(\mathbf{k})
$$

with $d_{\mu}(\mathbf{k})=\left(m_{\mathbf{k}},-2 \lambda \sin k_{x},-2 \lambda \sin k_{y},-2 \lambda \sin k_{z}\right)$. The actual form of our $\gamma$ matrices is apparent by comparing Eq.A1) to (9) but our result for $\theta$ is independent of any particular representation, as long as the matrices satisfy the requisite commutation relation. In fact in the subsequent calculations it will be advantageous to use a different representation of the Clifford algebra, obtained by a uniform rotation by angle $\pi / 2$ around the $\tau_{1}$ axis: we use $\gamma_{0}=\tau_{1} \otimes \mathbb{1}$ and $\gamma_{i}=\tau_{2} \otimes \sigma_{i}, i=1,2,3$.

In this representation the two normalized negativeenergy eigenstates of $\mathcal{H}_{\mathbf{k}}$ can be written as

$$
\begin{aligned}
& \psi_{1}=\left(-d_{1}+i d_{2}, d_{3}-i d_{0}, 0, i E\right)^{T} / \sqrt{2} E \\
& \psi_{2}=\left(d_{3}+i d_{0}, d_{1}+i d_{2},-i E, 0\right)^{T} / \sqrt{2} E
\end{aligned}
$$

where $E=\left(\sum_{\mu} d_{\mu}^{2}\right)^{1 / 2}$, and from now on we suppress the momentum dependence of all quantities. The eigenstates $\psi_{1}$ and $\psi_{2}$ above are degenerate and we are thus free to choose any (orthogonal) linear combination of these. Such a change of basis corresponds to a gauge transformation on $\mathcal{A}$. When evaluating $\theta$ using Eq. (13) one must keep in mind that the integrand is not gauge invariant while the integral taken over the BZ is gauge invariant modulo $4 \pi^{2}$. This property reflects the $Z_{2}$ nature of the topological invariant $\nu_{0}$ that underlies the physics of a STI.

Using the eigenstates given in Eq. A2 we find the Berry connection to be of the form $\mathcal{A}_{i}=\mathbf{n}_{i} \cdot \boldsymbol{\sigma}$, where $\boldsymbol{\sigma}$ is a vector of Pauli matrices and the components of vector $\mathbf{n}_{i}$ read

$$
\begin{aligned}
& n_{1 i}=D_{0} \partial_{i} D_{1}-D_{1} \partial_{i} D_{0}+D_{3} \partial_{i} D_{2}-D_{2} \partial_{i} D_{3}, \\
& n_{2 i}=D_{3} \partial_{i} D_{1}-D_{1} \partial_{i} D_{3}+D_{2} \partial_{i} D_{0}-D_{0} \partial_{i} D_{2}, \\
& n_{3 i}=D_{2} \partial_{i} D_{1}-D_{1} \partial_{i} D_{2}+D_{0} \partial_{i} D_{3}-D_{3} \partial_{i} D_{0},
\end{aligned}
$$

with $D_{\mu}=d_{\mu} / \sqrt{2} E$. After substituting these into Eq. (13) a tedious but ultimately straightforward calculation leads to the expression

$$
\theta=-\frac{1}{2 \pi} \int_{\mathrm{BZ}} d^{3} k \epsilon^{\alpha \beta \mu \nu} \frac{d_{\alpha} \partial_{1} d_{\beta} \partial_{2} d_{\mu} \partial_{3} d_{\nu}}{E^{4}} .
$$

Using the values of $d_{\mu}$ for our model given below Eq. (A1), one obtains a complicated integrand in terms of trigonometric functions. Although the value of the integral is guaranteed to be either 0 or $\pi$ in practice it is not obvious how to perform the required three-dimensional integration. However, it is clear from the structure of the integrand in (A3) that in the limit $\left|m_{\mathbf{k}}\right| \ll \lambda$ contributions to the integral come only from the vicinity of the 8 Dirac points. We evaluate these 8 contributions separately by linearizing $d$ 's as $d_{\mu}^{\ell}(\mathbf{k})=\left(m_{\ell}, v_{x}^{\ell} k_{x}, v_{y}^{\ell} k_{y}, v_{z}^{\ell} k_{z}\right)$ and obtain

$$
\theta^{\ell}=-\frac{1}{2 \pi} \int d^{3} k \frac{m_{\ell} v_{x}^{\ell} v_{y}^{\ell} v_{z}^{\ell}}{\left(4 \lambda^{2} k^{2}+m_{\ell}^{2}\right)^{2}} .
$$

An elementary evaluation then yields the result quoted in Eq. (14).
1 R.D. Peccei and H.R. Quinn, Phys. Rev. Lett. 38, 1440 (1977); Phys. Rev. D 16, 1791 (1977).
2 S. Weinberg, Phys. Rev. Lett. 40, 223 (1978).

${ }^{3}$ F. Wilczek, Phys. Rev. Lett. 40, 279 (1978). 
${ }^{4}$ L.D. Duffy and K. van Bibber, New J. Phys. 11, 105008 (2009).

${ }^{5}$ L.D. Duffy et al. Phys. Rev. D 74, 012006 (2006).

6 Axions: Theory, Cosmology, and Experimental Searches, Ed. by R. Beig, W. Beiglböck and W. Domcke (Springer, 2008).

7 L. Fu, C.L. Kane, and E.J. Mele, Phys. Rev. Lett. 98 106803 (2007).

8 J.E. Moore and L. Balents, Phys. Rev. B 75 121306(R) (2007).

9 L. Fu and C.L. Kane, Phys. Rev. B 76, 045302 (2007).

10 H. Zhang, C.-X. Liu, X.-L. Qi, X. Dai, Z. Fang, and S.C. Zhang, Nature Phys. 5, 438 (2009).

11 D. Hsieh et al., Nature 452, 970 (2008).

${ }^{12}$ Y. Xia et al., Nature Phys. 5, 398 (2009).

13 Y.L. Chen et al., Science 325, 178 (2009).

14 X.-L. Qi, T.L. Hughes, and S.-C. Zhang, Phys. Rev. B 78, 195424 (2008).

15 A.M. Essin, J.E. Moore, D. Vanderbilt, Phys. Rev. Lett. 102, 146805 (2009).

16 Z. Wang, X.-L. Qi and S.-C. Zhang, New J. Phys. (to appear, arXiv:0910.5954).

17 R. Li, J. Wang, X. Li and S.-C. Zhang, Nature Phys. 6, 284-288 (2010).

18 E. Witten, Phys. Lett. B 86, 283 (1979).

19 I. Affleck, Phys. Rev. Lett. 57, 1048 (1986).

20 B. Seradjeh, J.E. Moore, and M. Franz, Phys. Rev. Lett.
103, 066402 (2009).

21 F. Wilczek, Phys. Rev. Lett. 58, 1799 (1987).

22 E. Fradkin, E. Dagotto, and D. Boyanovsky, Phys. Rev. Lett. 57, 2967 (1986).

23 H.-M. Guo and M. Franz, Phys. Rev. Lett. 103, 206805 (2009).

24 D.A. Pesin and L. Balents, Nature Phys. 6, 376-381 (2010).

25 P. Hosur, S. Ryu, and A. Vishwanath, Phys. Rev. B 81, 045120 (2010).

${ }^{26}$ Magnetic monopoles, Y.M Shnir (Springer, 2005).

27 K.A. Milton, Rep. Prog. Phys. 69, 1637 (2006).

28 R. Jackiw and C. Rebbi, Phys. Rev. D 133398 (1976).

29 J. Goldstone and F. Wilczek, Phys. Rev. Lett. 47, 986 (1981).

30 W. P. Su, J. R. Schrieffer, and A. J. Heeger, Phys. Rev. Lett. 42, 1698 (1979).

31 C. Castelnovo, R. Moessner, and S.L. Sondhi, Nature 451, 42 (2007).

32 T. Fennell et al., Science 326, 415 (2009).

33 D.J.P. Morris et al., Science 326, 411 (2009).

34 S.T. Bramwell et al., Nature 461, 956 (2009).

35 V.J. Goldman and B. Su, Science 267, 1010 (1995).

36 R. De-Picciotto et al., Nature 389, 162 (1997).

37 R. Yu, W. Zhang, H.-J. Zhang, S.-C. Zhang, X. Dai and Z. Fang, arXiv:1002.0946 